\title{
CYCLIC HOMOLOGY OF ALGEBRAS
}


This page is intentionally left blank 


\title{
CYCLIC HOMOLOGY OF ALGEBRAS
}

\author{
Peter Seibt \\ CNRS, Centre de Physique Theorique \\ Marseille, France
}


Published by

World Scientific Publishing Co. Pte. Ltd.

P.O. Box 128, Farrer Road, Singapore 9128

U. S. A. office: World Scientific Publishing Co., Inc.

687 Hartwell Street, Teaneck NJ 07666, USA

Library of Congress Cataloging-in-Publication data is available.

\section{CYCLIC HOMOLOGY OF ALGEBRAS}

Copyright @ 1987 by World Scientific Publishing Co Pte Ltd.

All rights reserved. This book, or parts thereof, may not be reproduced in any form or by any means, electronic or mechanical, including photocopying, recording or any information storage and retrieval system now known or to be invented, without written permission from the Publisher.

ISBN $9971-50-468-5$

$9971-50-470-7 \mathrm{pbk}$

Printed in Singapore by Utopia Press. 
To Sebastian, Julie

Anna and willy

छิ

పே 
This page is intentionally left blank 
Introduction.

\section{Chapter I. CYCLIC (CO)HOMOLOGY AND HOCHSCHILD} (CO)HOMOLOGY.

I.1 Preliminaries: Spectral Sequences. $\quad 1$

1.1.1 Filtered Complexes and Exact Couples. 1

I.1.2 The Spectral Sequence associated with an Exact Couple. 3

1.1.3 Convergence of a Spectral Sequence. 7

1.1.4 Double Complexes and their Spectral Sequences. 12

1.2 Cyclic (Co)homology and Hochschild (Co)homology. 17

I.2.1 The Double Complex $C(\mathrm{~A}) . \quad 17$

1.2.2 The Cyclic Homology of an Associative Algebra. 20

1.2.3 Generalities about Mixed Complexes. 23

1.2.4 Cyclic Homology and Hochschild Homology. 32

I.2.5 Nonunital and Reduced Cyclic Homology. 41

I.2.6 Cyclic Cohomology. 58

1.2.7 Morita-Invariance of Hochschild Homology and
of Cyclic Homology.

$\begin{array}{ll}\text { Comments on Chapter I. } & 84\end{array}$

$\begin{array}{ll}\text { References to Chapter I. } & 85\end{array}$

Chapter II. PARTICULARITIES IN CHARACTERISTIC ZERO. 86

II.1 Relation to de Rham Theory. 86

II.1.1 A First Approach: Noncommutative de Rham Complexes. 86

11. 1.2 Cyclic Homology and de Rham Cohomology of Commutative Algebras. 
viii

$\begin{array}{ll}\text { II.2 Relation to Lie Theory. } & 121\end{array}$

11.2.1 Preliminaries around Invariant Theory. 121

II.2.2 Cyclic Homology and the Lie Algebra Homology of Matrices.

$\begin{array}{lr}\text { Comments on Chapter II. } & 156\end{array}$

$\begin{array}{ll}\text { References to Chapter II. } & 157\end{array}$

$\begin{array}{lr}\text { Further references. } & 158\end{array}$

$\begin{array}{lr}\text { List of symbols and notations. } & 159\end{array}$

$\begin{array}{lr}\text { Index. } & 160\end{array}$ 
Introduction.

These lectures are an extended version of my contribution to a seminar on cyclic cohomology, held at the University of Marseille Luminy, in 1985. They are essentially based on a paper of J.L. Loday and D. Quillen: Cyclic homology and the Lie algebra homology of matrices (Comment: Math. Helvetici, 59 (1984), 565-591), and contain also ideas and results of $M$. Karoubi and $C$. Kassel.

The exposition is purely algebraic, according to my own background, and thus concentrates rather on cyclic homology (than on cohomology), the former being a more natural starting point for an algebraist. But many of the leading ideas of the theory, more apparent in cohomology, come from topology and differential geometry, in the language of operator algebras. Thus it should be clear that this is not an introduction to cyclic (co)homology, but only the attempt to single out the basic algebraic facts and techniques of the theory. The reader who wants more motivations should imperatively consult the fundamental article of Alain Connes: Noncommutative differential geometry, I.H.E.S. Pub1. Math. vol. 62 (1985), 41-144.

The lectures are organized in two chapters.

The first chapter deals with the intimate relation of cyclic theory to ordinary Hochschild theory, which is at least not surprising by the parallel definition of both theories. There are some important quasiisomorphisms, proving the equivalence of different approaches to cyclic homology, and spectral sequence techniques are convenient to establish these facts. Thus a comforting paragraph on spectral sequences opens the exposition. Fortunately, I could already take in account the extremely elegant mixed complex approach to cyclic homology of D. Burghelea which streamlines a lot of arguments. The first climax is the fundamental long exact sequence

$\cdots \rightarrow \mathrm{H}_{n}(A) \rightarrow \mathrm{HC}_{\mathrm{n}}(A) \rightarrow \mathrm{HC}_{\mathrm{n}-2}(A) \rightarrow \mathrm{H}_{\mathrm{n}-1}(A) \rightarrow \cdots$

relating the Hochschild homology groups and the cyclic homology groups (analogously in cohomology), a cornerstone for all structural transmission between both theories. Nomalized mixed Hochschild complexes and reduced theory are treated in order to invest conveniently differential ideas: Our operator $B$ becomes a good candidate for a non- 
commutative outer derivative. Finally, Morita-invariance of Hochschild homology and of cyclic homology are treated, following closely an exposition of $\mathrm{K}$. Igusa.

The second chapter deals with cyclic homology as a typical characteristic zero theory. First, its relation to de Rham cohomology is considered. It comes out that (noncommutative) de Rham cohomology in the sense of $M$. Karoubi can be embedded in (reduced) cyclic homology. For smooth commutative algebras this can be made more precise by a sort of inverse limit constellation, which is formulated via a decomposition of cyclic homology into ordinary de Rham cohomology:

$H C_{n}(A)=\Omega^{n} / d \Omega^{n-1} \oplus H_{D R}^{n-2}(A) \oplus H_{D R}^{n-4}(A) \oplus \cdots$

This result of $J . I$. Loday and $D$, Quillen has a dual version in continuous cyclic cohomology, due to $A$. Connes (with $A=C^{\infty}(x, c)$, the $\mathbb{c}$-algebra of smooth complex-valued functions on a compact manifold $X$ ). The final sections of the second chapter deal with cyclic homology as "additive $\mathrm{K}$-theory", in the following sense: For an associative algebra $A$ over a field $k$ of characteristic zero, cyclic homology $\mathrm{HC}_{*}(\mathrm{~A})$ is, up to a dimension shift, isomorphic to the space of primitive elements Prim $H_{*}(g l(A))$ of the Lie algebra homology of $g I(A)=\lim _{\rightarrow} g I_{r}(A)$. This result should be appreciated in the light of $\mathrm{D}$. Quillen's "multiplicative"version: Rational algebraic K-theory $K_{*}(A) \otimes Q$ identifies with Prim $H_{*}(G L(A), Q)$, the primitive part of (discrete) group homology of $G L(A)=\lim _{\rightarrow} G_{r}(A)$. I have to admit two important algebraic omissions: First, I did not treat products (essential in cohomology, which I neglected $a$ bit) and Künneth-formulas (since I got afraid of the costructure invasion). Then, which is perhaps more serious, I did not treat the relations to algebraic $\mathrm{k}$-theory via Chern-characters. On a certain level of arguments this motivates half of the existence of the theory ("create a range for invariants"), but whenever you make the first step towards topology and geometry ...

A few words about the use of spectral sequences in these lectures. Me only need them in order to establish some fundamental quasi-isomorphisms, by an approximation argument. This could also be done via explicit and involved calculations. But, since we aim at furnishing the necessary material for further reading, there is no reason to avoid spectral sequence techniques (look at the literature!). At any rate, there is a coherent approach to the basic skeleton of the theory, avoiding all spectral sequence arguments. You begin with I.2.3; then you define cyclic homology of a unital associative k-algebra $A$ as the cyclic homology of the mixed complex $C(A)$ (cf. I.2.4.2). You get all of the 
material from I.2.4.6 to I.2.4.12. Reduced theory remains unchanged (I.2.5.1 to I.2.5.13). Cyclic cohomology is treated analogously. As to Morita-invariance, the spectral sequence argument in I.2.7.9 is easily replaced by a direct reasoning. de Rham theory (II.1) is already clean. Thus you cover rapidly all of the basic material. It should be pointed out, however, that the equivalence of the different approaches to cyclic (co)homology is an essential part of its handiness. Finally, I would like to express my thanks to all those who helped me to finish these lectures: first of all, to Daniel Kastler, whose stimulating enthousiasm for the subject and clever support (on many levels) pushed me across this experience. Then, to Joachim Cuntz and Georges zeller-Meier, who taught me the essentials of the subject, and finally to Philippe Blanchard, Sergio Doplicher, Rudolph Haag and Daniel Testard, whose hospitality and interest at different stages of the work I shall never forget.

This paper was written while the author was guest of the Research Center Bielefeld-Bochum-Stochastics (BiBos) at the University of Bielefeld. I would like to express my thanks for its kind hospitality. Thanks also to Mrs. Aoyama-Potthoff for the excellent and competent typewriting.

Rome, May 1986

Peter Seibt 